ARTIGO ORIGINAL

\title{
PERFIL EPIDEMIOLÓGICO DOS CASOS DE DOENÇA INFLAMATÓRIA INTESTINAL EM PACIENTES PEDIÁTRICOS NA REGIÃO NORTE DO BRASIL ENTRE 2010 E 2019
} EPIDEMIOLOGICAL PROFILE OF CASES OF INFLAMMATORY BOWEL DISEASE IN PEDIATRIC PATIENTS IN NORTHERN BRAZIL BETWEEN 2010 AND 2019

Aline Rangel de Souza ${ }^{1}$; Débora Sousa Negreiros ${ }^{1}$; Ingrid Fernandes de Souza ${ }^{1}$; Euber Joe Jurado Martinez ${ }^{1}$; Evandro Leite Bitencourt ${ }^{2}$

\begin{abstract}
RESUMO
Introdução: A doença inflamatória intestinal (DII) compreende a doença de Crohn (DC) e a Colite ulcerativa (CU) e pode ser diagnosticada na infância e na adolescência em 25\% dos casos. Objetivo: Estudar a distribuição dos casos de DII em crianças e adolescentes na faixa de menor de 1 a 14 anos de idade entre os anos de 2010 e 2019 na região Norte do Brasil. Métodos: Um estudo epidemiológico descritivo realizado com dados obtidos por meio do DATASUS. Foram pesquisados dados da Região Norte no período de 2010 a 2019 e analisados os valores relativos às internações, estratificados conforme faixa etária, sexo, cor/etnia por DII nos estados supracitados. Resultados: O Estado do Pará, com $66,86 \%$ (440) dos casos registrados, apresentou um valor expressivamente maior que os demais. Houve um aumento gradativo da incidência de DII até 2012, havendo a partir de 2013 uma queda progressiva no número de casos. No geral, $54,25 \%$ dos pacientes foram do sexo masculino e $45,74 \%$ do sexo feminino. Conclusão: Apesar da diminuição dos casos com base nos dados coletados, essa doença possui um impacto na qualidade de vida e morbimortalidade desses pacientes. Sendo assim, é necessário ações de atenção básica para a prevenção dessa enfermidade
\end{abstract}

Palavras-chave: Pediatria, Doença de Crohn, Colite Ulcerativa
Souza IF, Mação: Souza AR, Negreiros DS, Perfil epidemiológico dos casos de Doença Inflamatória Intestinal em pacientes pediátricos na região Norte do Brasil entre 2010 e 2019 . Revista de Patologia do Tocantins, 10(4):.

\section{Instituição:}

${ }^{1}$ Acadêmico (a) de Medicina, Universidade Federal do Tocantins, Palmas, Brasil.

${ }^{2}$ Graduado em Química; Mestre em Química; Acadêmico Medicina, Universidade Federal do Tocantins, Palmas, Brasil; Pesquisador Instituto Médico Legal do Tocantins (IML/TO), Palmas, Brasil.

Autor correspondente: Aline Rangel de Souza; aline.rangel@mail.uft.edu.br

Editor: Carvalho A. A. B. Medicina, Universidade Federal do Tocantins, Brasil.

Publicado: 18 de outubro de 2020.

Direitos Autorais: (c) 2020 Souza et al. Este é um artigo de acesso aberto que permite o uso, a distribuição e a reprodução sem restrições em qualquer meio, desde que o autor original e a fonte sejam creditados.

Conflito de interesses: os autores declararam que não existem conflitos de interesses.

\begin{abstract}
Introduction: Inflammatory bowel disease (IBD) comprises Crohn's disease (CD) and ulcerative colitis (CU) and can be diagnosed in childhood and adolescence in $25 \%$ of cases. Objective: To study the distribution of IBD cases in children and adolescents aged between 1 and 14 years old between the years 2010 and 2019 in the northern region of Brazil. Methods: A descriptive epidemiological study conducted with data obtained through DATASUS. Data from the North Region were searched from 2010 to 2019 and the values related to hospitalizations were analyzed, stratified according to age group, sex, color/ ethnicity by IBD in the above-mentioned states. Results: The State of Pará, with $66.86 \%$ (440) of the registered cases, presented a value significantly higher than the others. There was a gradual increase in the incidence of IBD until 2012, with a progressive drop in the number of cases starting in 2013 . Overall, $54.25 \%$ of the patients were male and $45.74 \%$ female. Conclusion: Despite the decrease in cases based on the data collected, this disease has an impact on the quality of life and morbidity and mortality of these patients. Therefore, it is necessary to provide primary care actions to prevent this disease
\end{abstract}

Keywords: Pediatrics, Crohn disease, Ulcerative Colitis. 


\section{INTRODUÇÃO}

A doença inflamatória intestinal (DII) é uma das doenças crônicas mais comuns e com maior impacto na gastroenterologia pediátrica. ${ }^{1}$ As DIls, que incluem a Doença de Crohn (DC) e Colite Ulcerativa (CU), são manifestações crônicas e heterogêneas, induzidas pela interação de fatores ambientais, genéticos, da microbiota intestinal e imunológicos, que evoluem causando dano intestinal progressivo ao longo do tempo. ${ }^{2,3,4,5,6}$ Podem ser diagnosticadas na infância e na adolescência em $25 \%$ dos casos. Cerca de $15 \%$ dos diagnósticos de DII permanecem como colite não definida (CND) por algum tempo na evolução da doença. ${ }^{7,8}$ Por se tratar de uma doença crônica, tendem a ser progressivas e afetar vários aspectos do cotidiano dos doentes comprometendo a qualidade de vida dos mesmos., ${ }^{9,10}$

Estima-se que mais de um milhão de pessoas nos Estados Unidos da América (EUA) tenha a DII e cerca de $10 \%$ destes pacientes são crianças. A incidência de DII em crianças está aumentando. ${ }^{11}$ No Brasil, a DIl persiste como uma importante causa de morbimortalidade em pacientes pediátricos. ${ }^{12,13} \mathrm{Em}$ geral, as crianças apresentam quadros iniciais mais graves e extensos da doença, maior prevalência de manifestações extra intestinais e importante comprometimento nutricional, além de particularidades como atraso do crescimento e do desenvolvimento, quando comparadas aos adultos. Em vista disso, o diagnóstico precoce e preciso é determinante para iniciar o tratamento e evitar complicações. Recomenda-se a realização de imagens de intestino delgado em todos os casos suspeitos de DIl no diagnóstico. 6,11,13

Em vista das particularidades apresentadas pelos pacientes, ocorrem desafios em cada etapa da doença. Não há um exame específico para se firmar o diagnóstico de doença de Crohn ou colite ulcerativa. Portanto, o diagnóstico das doenças inflamatórias intestinais (DII) na idade pediátrica deve ser baseado na combinação da história clínica, exame físico, exames laboratoriais, endoscopia digestiva alta e ileocolonoscopia com histologia e método diagnóstico por imagem do intestino delgado. ${ }^{14}$

Entre os exames radiológicos utilizados na avaliação/diagnóstico de DC, podemos citar a radiologia convencional/contrastada, ultrassonografia, tomografia computadorizada (entero-TC) e ressonância magnética (entero-RM). Imagem do intestino delgado é essencial para mapear a localização da doença, avaliar severidade, identificar complicações como as fistulas, abscessos e estenoses intestinais. ${ }^{15,16}$

Infelizmente, as condutas terapêuticas pediátricas são, em sua maioria, uma extrapolação do que é conhecido e disponível para adultos com DII. O tratamento medicamentoso para a DII na criança e adolescente deve ser individualizado, baseado primeiramente na diferenciação entre DC ou CU, determinação da localização e extensão da lesão, presença ou não de manifestações extraintestinais e o estado nutricional do paciente. As drogas comumente utilizadas são os aminossalicilatos (Sulfassalazina, Mesalazina, Asacol $^{\circledR}$, Asalit ${ }^{\circledR}$ e Pentasa $^{\circledR}$ ), corticoides (Prednisona, Prednisolona, Metilprednisolona e Hidrocortisona), imunossupressores (Azatioprina, 6-mercaptopurina, Metotrexato, Ciclosporina e Tacrolimo) e terapia biológica
(Infliximabe Remicade ${ }^{\circledR}$, Adalimumabe e Humira ${ }^{\circledR}$ ). Sendo que, esses medicamentos são garantidos pelo Sistema Único de Saúde (SUS). ${ }^{1,17,18,19}$

\section{OBJETIVOS}

Tendo em vista a importância desse assunto para a pediatria, o presente artigo objetiva estudar a distribuição dos casos de DIl em crianças e adolescentes na faixa de menor de 1 a 14 anos de idade entre os anos de 2010 e 2019 na região Norte do Brasil.

\section{MÉTODO}

Realizou-se um estudo epidemiológico descritivo acerca das internações por doença de Crohn e por colite ulcerativa na Região Norte, por unidade da federação dos estados do Acre (AC), Amapá (AP), Amazonas (AM), Pará (PA), Rondônia (RO), Roraima (RR) e Tocantins (TO), referentes ao período de janeiro de 2010 a dezembro de 2019. Os dados foram obtidos a partir do Departamento de Informática do Sistema Único de Saúde (DATASUS) por meio da ferramenta de pesquisa TABNET, sendo a fonte original o Sistema de Informações Hospitalares do SUS (SIH / SUS). As informações coletadas foram estratificadas por estado, ano, sexo, cor/ etnia e a faixa etária de menor de 1 ano a 14 anos. Os dados foram tabulados em planilha Excel, realizado uma análise descritiva dos achados mais significativos, apresentando-se em tabelas. A pesquisa não possui conflito de interesses

\section{RESULTADOS}

Observa-se que o número de internações na Região Norte durante o período foi de 638 casos, apresentando um aumento gradativo da incidência de DII até 2012, com um pico de incidência geral de 127 casos, havendo a partir de 2013 uma queda progressiva com pequenas oscilações no número de casos da doença, com um pequeno aumento em 2019. Desses, o estado do Pará, com 66,86\% (440) dos casos registrados, apresentou um valor expressivamente maior que os demais, sendo seguido por Acre, 14,13\% (93), Amazonas, $8,05 \%$ (53), e com o menor número está Roraima 0,91\% (6) (Figura 1).

Figura 1. Número de internações por Doença de Crohn e colite ulcerativa na região Norte por unidade federativa de 2010 e 2019, na faixa etária de menor de 1 ano a 14 anos.

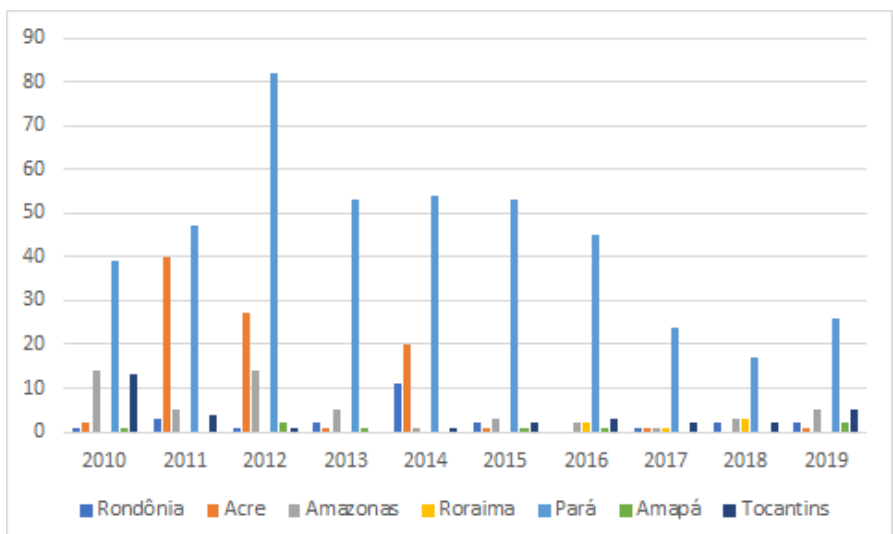

Fonte: DATASUS ${ }^{21}$. 
Identifica-se que a maior incidência total ocorreu na faixa etária de 1 a 4 anos com 37,38\% (246) dos casos e a menor incidência na faixa etária de menor de 1 ano com $17,78 \%$ (117) dos casos (Figura 2). Além disso, evidenciou-se esse padrão de faixa etária nos Estados.

Figura 2. Número casos por Doença de Crohn e colite ulcerativa na região Norte por faixa etária de menor de 1 ano a 14 anos de 2010 e 2019.

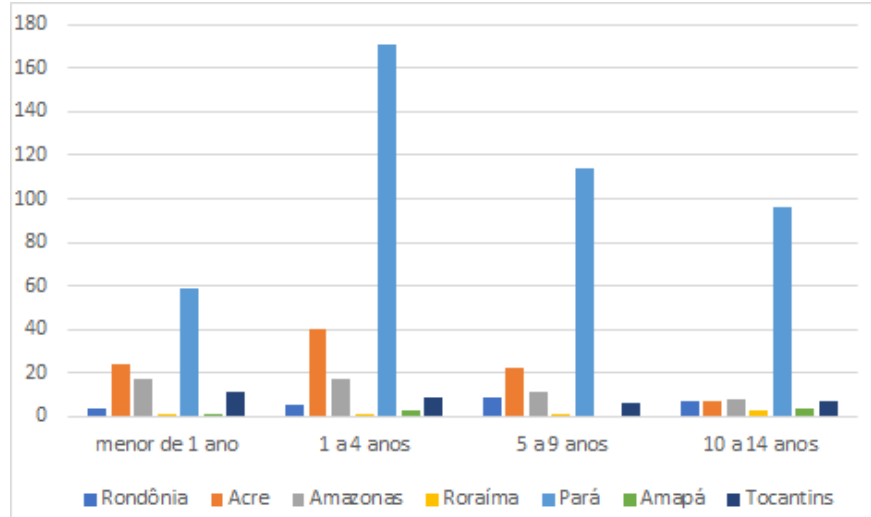

Fonte: DATASUS ${ }^{21}$.

Na categorização de sexo, o número de casos foi maior em pessoas do sexo masculino com um total de 357 $(54,25 \%)$ e no sexo feminino com um total de 301 (Figura 3 ). Em cinco dos sete estados, o sexo masculino teve maior incidência, sendo que em Roraima ocorreu com maior frequência no sexo feminino e em Amapá igual quantidade de casos em ambos os sexos.

Figura 3. Número de casos de Doença de Crohn e colite ulcerativa por sexo na região Norte do Brasil de 2010 e 2019, na faixa etária de menor de 1 ano a 14 anos.

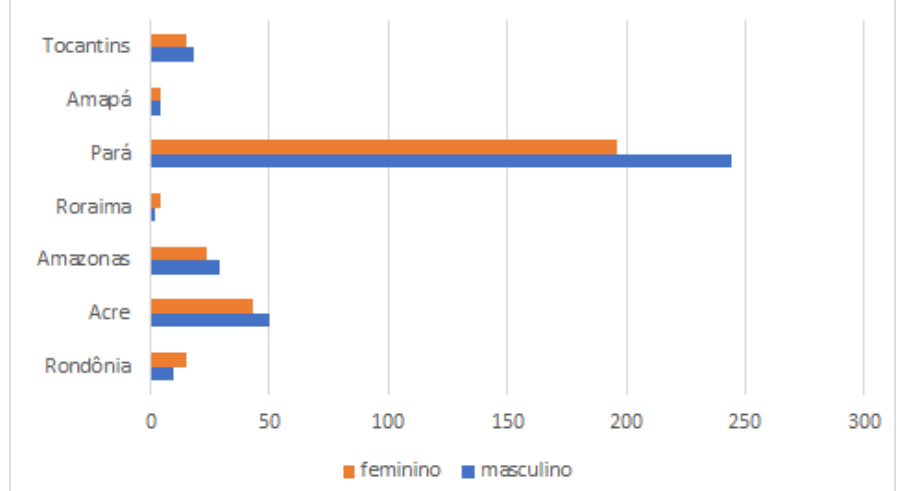

Fonte: DATASUS ${ }^{21}$

Percebe-se que $58,2 \%$ (383) dos casos não apresentam a informação de cor/ etnia. Os $41,8 \%$ (275) dos casos que apresentam essa informação, 90,18\% ocorrem em pardos, $5 \%$ em brancos, 3,27 \% em indígenas e 1,8\% em amarelos (Figura 4).

Figura 4. Número de casos de Doença de Crohn e colite ulcerativa por cor/ etnia na região Norte do Brasil de 2010 e 2019, na faixa etária de menor de 1 ano a 14 anos

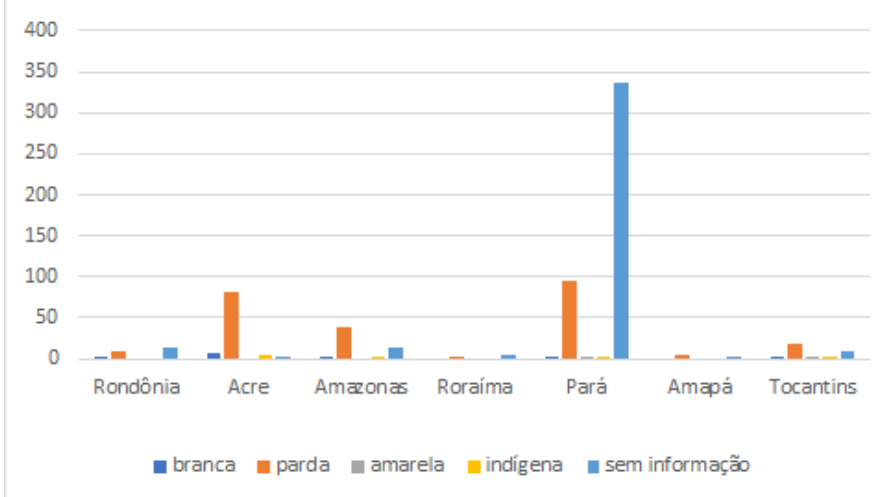

Fonte: DATASUS ${ }^{21}$

\section{DISCUSSÃO}

Estudos revelam que países desenvolvidos apresentaram declínio das DII. No entanto, países em desenvolvimento demonstram aumento do número de $\operatorname{casos}^{22}$. No Brasil, a prevalência da doença encontra-se na região Sul e Sudeste, e está relacionada a qualidade de vida, como padrão socioeconômico e saneamento básico, ocasionando uma modificação da microbiota intestinal $\left.\right|^{6,22,23}$. Neste estudo observou um declínio no número de casos, sendo contrário aos achados da literatura. De certo, a prevalência real dos casos seja subestimada por falta de notificações dos casos.

Sabe-se que a DC e a CU podem apresentar epidemiologias variáveis. Assim, um estudo em um hospital de Santa Catarina, observou maior prevalência em mulheres com DC e em homens com a $\mathrm{CU}^{22}$. No entanto, com predomínio do sexo feminino para as DII. Na região Norte os dados não estão de acordo com a literatura. Observou maior prevalência dos casos no sexo masculino, tendo apenas o estado de Roraima com prevalência do sexo feminino.

Quanto a idade, ainda há poucos estudos que abrangem idades inferiores a 19 anos, tendo maior prevalência da doença entre 25 a 60 anos $^{22,24}$. Porém, 25\% dos pacientes realizam o diagnóstico de DC na infância, e 15 a $40 \%$ dos pacientes realizam o diagnóstico de RC com menos de 20 anos $^{6,24,25}$. Já quanto a etnia, observou uma predominância do grupo que se declarou branco. Estudos apontam que pacientes com descendentes brancos apresentaram maior chance de ter a doença ${ }^{6,24}$. Porém, na análise dos dados da região norte observou maior predomínio do grupo de pardos. Deste modo, é importante ressaltar a necessidade de informar dados mais detalhados e deve ser mais investigado na prática clínica, para maior diagnóstico e análise das DII na região norte.

\section{CONCLUSÃO}

Os dados encontrados por este estudo, portanto, mostraram que o perfil epidemiológico dos casos de Doenças Inflamatória Intestinal em pacientes pediátricos na região Norte no período de 2010 a 2019 é composto por indivíduos do sexo masculino da raça parda na faixa etária de 1 a 4 anos, sendo o Pará o estado com mais casos registrados. Outrossim, 
evidenciaram uma prevalência dos casos em áreas endêmicas para as diarreias e gastroenterite e uma queda gradual a partir de 2013. Apesar da diminuição dos casos com base nos dados coletados, essa afecção possuí um impacto na qualidade de vida e morbimortalidade desses pacientes, principalmente aqueles com baixo nível sócio econômico e dificuldade de acesso a centros especializados de saúde. Sendo assim, é necessário ações de atenção básica e planejamento de políticas públicas visando a prevenção dessa enfermidade, minimizando os danos potenciais que essa patologia possa causar às crianças e adolescentes

\section{REFERÊNCIAS}

1. Dewulf N.L.S. Investigação sobre a adesão ao tratamento medicamentoso em pacientes com doenças inflamatórias intestinais. 2005. 99f. Dissertação de Mestrado - Faculdade de Medicina de Ribeirão Preto, Universidade de São Paulo, Ribeirão Preto, 2005.

2. Cambui, Y.R.S.; Natalil, M.R.M. Doenças inflamatórias intestinais: revisão narrativa da literatura. Rev Ciênc Méd, Sorocaba, v.17, n.3, p.116-119, 2015.

3. Figueiredo, A.A.; Mota, J.A.C.; Penna, F.J. Silva, G.A.P. Colite ulcerativa inespecífica em crianças e adolescentes: análise de casuística. Rev Bras Saúde Matern Infant, Recife, v.4, n.3, p. 309-315, 2004.

4. Nóbrega, V.G et al. The onset of clinical manifestations in inflammatory bowel disease patients. Arq Gastroenterol, v.55, n.3, p. 290-295, 2018.

5. Oliveira, F.M.; Emerick, A.P.C.; Soares, E.G. Aspectos epidemiológicos das doenças intestinais inflamatórias na macrorregião de saúde leste do Estado de Minas Gerais. Ciênc. saúde coletiva [online], v.15, s.1,pp.1031-1037, 2010.

6. Sdepanian, VL et al. Doença inflamatória intestinal em Pediatria. São Paulo: Editora Mazzoni, 2019.

7. Costa, S. et al. Qualidade de vida na doença inflamatória intestinal pediátrica: validação do questionário IMPACT IIIC para a população portuguesa. Acta Pediatr Port, v.43, n.5, p.198-201, 2012

8. Rodrigues, M. Diagnóstico da Doença Inflamatória Intestinal na Criança e Adolescente. International Journal of Nutrology, v.10, n.1, 281-293, 2017.

9. Kleinubing-Junior, $H$. et al. Perfil dos pacientes ambulatoriais com doenças inflamatórias intestinais. $A B C D$, Arq. bras. cir. dig. [online], v.24, n.3, p.200-203, 2011.

10. Souza, M.M. Qualidade de vida de pacientes portadores de doença inflamatória intestinal. ActaPaul Enferm, São Paulo, v. 24, n.4, p. 479-484, 2011.

11. Marques, M.L.A.; Patrício, M.P.F. Manifestações extra intestinais de espectros da doença inflamatória intestinal em crianças e adolescentes: artigo de revisão. Ver Med UFC, v.59, n.1, p. 44-52, 2018

12. Camargo-Cruz P.E.A. Distribuição espacial-temporal das internações por doenças infecciosas intestinais no estado de São Paulo, para a faixa etária de 5 a 14 anos, entre 2001 e 2010. Atas de Saúde Ambiental (São Paulo, online), v. 5, p. 85-104, 2017.

13. Melo, M.C.B. de et al. Doença inflamatória intestinal na infância. Rev Med Minas Gerais, v.26, s.2, p.35-44, 2016.

14. Levine A, Koletzko S, Turner D, et al; European Society of Pediatric Gastroenterology, Hepatology, and Nutrition. ESPGHAN revised porto criteria for the diagnosis of inflammatory bowel disease in children and adolescents. J Pediatr Gastroenterol Nutr, 2014, n.58, pp. 795-806.
15. Maltz R, Podberesky DJ, Saeed SA. Imaging modalities in pediatric inflammatory bowel disease. Curr Opin Pediatr, 2014, n.26, v.5, pp.590-596.

16. Cantarelli BCF et al. Avaliação da atividade inflamatória da doença de Crohn por métodos seccionais de imagem. Radiol Bras. 2020, n. 53, v.1, pp. 38-46.

17. Sdepanian VR. Junqueira JCF, Koda YKL. Doença Inflamatória Intestinal. In: Gastroenterologia Pediátrica: manual de condutas/coordenadora Vera Lucia Sdepanian. Ed Manole, 2010. p 81-93.

18. Brasil. Protocolos Clínicos e Diretrizes Terapêuticas - PCDT. Disponível em: http://www.saude.gov.br/protocolos-ediretrizes. Acesso em: 31 Ago 2020.

19. Associação brasileira de Colite Ulcerativa e Doença de Cronhn. Cartilha dos direitos e responsabilidades dos pacientes com Doença Inflamatória Intestinal. São Paulo: Editora Adenilde Bringel, 2016. Disponível em: https://www.abcd.org.br/noticias/cartilha-dos-direitos-eresponsabilidade-dos-pacientes-de-dii/. Acesso em: 01 Set 2020.

20. Santos, G.M. dos; Silva, L.R.; Santana, G.O. Repercussões nutricionais em crianças e adolescentes na presença de doenças inflamatórias intestinais. Rev. paul. pediatr. [online]. 2014, vol.32, n.4, pp.403-411.

21. Departamento de Informática do SUS [Internet]. Brasília (DF): Ministério da Saúde; 2020. Disponível em: http://datasus.saude.gov.br/informacoes-desaude/portalde-saude-cidadao.

22. Ferraz FB. Panorama Geral Sobre Doenças Inflamatórias Intestinais: imunidade e Suscetibilidade da Doença de Crohn e Colite Ulcerativa. Health Sci 2016;18(2):139-43.

23. Arantes JAV, Santos CHM, Delfino BM, et.al. Epidemiological profile and clinical characteristics of patients with intestinal inflammatory disease. J. coloproctol (rio j). $2017 ; 3$ 7(4):273-278

24. Gasparini RG. Incidência e Prevalência de Doenças Inflamatórias Intestinais no Estado de São Paulo - Brasil. Tese [Doutorado em Bases Gerais da Cirurgia] - Faculdade de Medicina, Universidade Estadual Paulista "Júlio de Mesquita Filho", 2018.

25. MELO MCB, et al. Doença Inflamatória Intestinal na Infância. Rev Med. Minas Gerais, 2016; 26 (Supl 2): S35S44. Disponível em: file:///C:/Users/marce/Downloads/v26s2a07.pdf 